\title{
Sistema de Monitoramento de Amplitude de Movimento Baseado em Redes de Sensores sem Fio Aplicado à Fisioterapia
}

\author{
Renan C. A. Alves ${ }^{1}$, Fabíola C. L. dos Santos ${ }^{2}$, Bruno T. de Oliveira ${ }^{1}$, Cíntia B. Margi ${ }^{1}$ \\ ${ }^{1}$ Laboratório de Arquitetura e Redes de Computadores \\ Departamento de Engenharia de Computação e Sistemas Digitais \\ Escola Politécnica - Universidade de São Paulo \\ São Paulo - SP - Brasil \\ ${ }^{2}$ Departamento de Fisioterapia, Fonoaudiologia e Terapia Ocupacional \\ Faculdade de Medicina - Universidade de São Paulo \\ São Paulo - SP - Brasil \\ \{renanalves, fasantos, brunotrevizan, cintia\}@usp.br
}

\begin{abstract}
Range of motion is an important parameter used to evaluate and during physical therapy treatment. The FlexMeter system provides real time joint angles measurement and visualization, as well as stored data analysis. The system is based on wireless sensor networks with accelerometer nodes. The physical therapist, through the system interface, monitors the session, writes observations, and also analyses the patient evolution through multiple sessions. Validation results show how the system use could ease the physical therapist work.
\end{abstract}

Resumo. A amplitude de movimento é um parâmetro importante utilizado na avaliação e durante o tratamento fisioterapêutico. O sistema FlexMeter permite a mensuração e a visualização de ângulos articulares em tempo real e a análise posterior a partir de dados armazenados. $O$ sistema baseia-se em redes de sensores sem fio com módulos sensores munidos de acelerômetros. A interface do sistema permite ao fisioterapeuta acompanhar a sessão, inserir observações, bem como analisar a evolução do paciente ao longo de múltiplas sessões. Os resultados da validação demonstraram como seu uso pode facilitar o trabalho do fisioterapeuta.

\section{Introdução}

As grandes evoluções da medicina costumam acompanhar as evoluções tecnológicas, caracterizando um processo multidisciplinar, envolvendo principalmente as ciências médicas e a engenharia. Um dos principais fatores deste processo de evolução são os instrumentos de mensuração, que permitem um monitoramento do estado de saúde do paciente, facilitando as decisões dos profissionais da área saúde. O advento da tecnologia da informação e a utilização dos sistemas de informação, possibilitaram uma análise mais refinada dos dados dos pacientes, bem como a manutenção de um histórico médico de cada indivíduo. No domínio de conhecimento da saúde, a fisioterapia mostra-se uma área carentes destes aplicativos e ferramentas.

A fisioterapia é uma ciência da saúde que estuda, previne e trata os distúrbios cinéticos funcionais intercorrentes em órgãos e sistemas do corpo humano, gerados por 
alterações genéticas, por traumas e por doenças adquiridas. Esta fundamenta suas ações em mecanismos terapêuticos próprios [COFFITO 2012].

A goniometria, ou mensuração dos ângulos, de articulações é uma tarefa comum a profissionais da área de fisioterapia, tratando-se de uma métrica importante para quantificar a evolução do quadro de seus pacientes [Marques 2003]. As ferramentas existentes requerem que o profissional interrompa a sessão para a obtenção de mensurações necessárias, e demandam considerável trabalho manual para o registro dos dados obtidos.

A adoção de sistemas de informação em conjunto com mecanismos automatizados de coleta dos dados, como Redes de Sensores sem Fio (RSSF), permite ao profissional concentrar seus esforços e atenção no paciente, focando na análise dos dados de evolução e na orientação dos exercícios. Ademais, a partir do armazenamento dos dados coletados é possível manter um histórico detalhado de cada tratamento.

As RSSF são comumente utilizadas em aplicações de monitoramento e podem ser definidas como uma classe especial de redes ad hoc de múltiplos saltos (MANETs multihop ad hoc networks), são redes sem fio que não possuem qualquer infra-estrutura fixa. Tipicamente, os nós participantes possuem baixa capacidade de processamento, pouca memória para armazenamento, sistemas de comunicação de baixa velocidade e largura de banda, e fonte de energia limitada [Culler et al. 2004].

O projeto apresentado visa conciliar engenharia e fisioterapia, permitindo a mensuração e a visualização de ângulos articulares em tempo real, por meio de RSSF, e a análise posterior a partir de dados armazenados. Através do estudo da evolução das medidas é possível construir um indicador da efetividade dos exercícios e de possíveis dificuldades encontradas pelos pacientes, permitindo o acompanhamento e a supervisão dos respectivos tratamentos. Assim, o trabalho almeja contribuir junto à área de fisioterapia no que diz respeito a sistemas de monitoramento, e para tanto descrever projeto, implementação e validação de um protótipo do sistema FlexMeter.

Este trabalho está organizado da seguinte forma. A Seção 2 trata dos trabalhos relacionados. A Seção 3 especifica e detalha as características de hardware e software do sistema FlexMeter, aqui apresentado. Em seguida, os resultados da validação de medidas e usabilidade do sistema são apresentados e discutidos na Seção 4, enquanto a Seção 5 apresenta as considerações finais e trabalhos futuros.

\section{Trabalhos Relacionados}

Os trabalhos relacionados discutidos nesta seção podem ser divididos em duas categorias, de acordo com sua proximidade a engenharia (seção 2.1) ou fisioterapia (seção seção 2.2).

\subsection{Redes de Sensores Sem Fio}

Uma família de aplicações de RSSF que se destaca é a de eHealth. Seu uso permite aplicações de monitoramento mais robustas, devido a diversos contextos pessoais que podem ser capturados e usados para prover melhores serviços de saúde, como emergência, cuidado com idosos, e auxílio a fisioterapia.

O projeto CodeBlue [Malan et al. 2004] possui aplicação em ambiente hospitalar, triagem de pacientes, entre outros. O projeto Mercury [Lorincz et al. 2009] é uma plata- 
forma wearable baseada em RSSF para análise de movimento de pacientes em tratamento de doenças neuromotoras, como Mal de Parkinson, epilepsia e derrame cerebral.

O monitoramento dos batimentos cardíacos do paciente e a sua postura em sessões de hidroterapia é proposto por [Silva et al. 2007]. A postura é monitorada através de ângulos rotacionais dos membros do corpo com sensores interligados com cabos. Os dados são enviados a estação base por um módulo sensor flutuante, de modo a evitar a comunicação sem fio subaquática. Tal trabalho tem como objetivo medir apenas a posição da coluna, e não do ângulo de movimento de outros membros.

A teoria para se realizar mensuração de ângulos com redes de sensores através do uso de acelerômetros, giroscópios e magnetômetros é descrita em [O'donovan et al. 2007]. Uma prova de conceito para medidas de ângulos de joelho e tornozelo é descrita em [O’Donovan and Ayer 2011], não mencionando a existência de um sistema de informação para auxiliar a análise dos dados obtidos.

\subsection{Instrumentação de Fisioterapia}

A amplitude de movimento $(\mathrm{ADM})^{1}$ é um importante parâmetro utilizado na avaliação e durante o tratamento fisioterapêutico. Muitas vezes a avaliação da amplitude do movimento faz parte da definição da propedêutica e do prognóstico de um indivíduo submetido a fisioterapia [Portney and Watkins 2000].

A ADM varia de indivíduo para indivíduo de acordo com a idade, sexo, prática de atividade física, presença ou ausência de disfunção e o grau de força muscular quando o indivíduo é submetido à avaliação da ADM ativa [Rothstein 1985]. A padronização da metodologia empregada é de extrema importância uma vez que pode evitar possíveis vieses.

Alguns instrumentos vêm sendo utilizados para tal mensuração. O mais utilizado é o goniômetro universal, por ser de fácil utilização e baixo custo, para a mensuração é necessário a utilização de pontos anatômicos determinados [Gajdosik and Bohannon 1987].

Outro instrumento é o flexímetro, cujo sistema pendular gravitacional possui indicação do ângulo produzida por efeito da gravidade, minimizando os erros de interpretação do eixo longitudinal correspondente. A escala angular foi desenhada com incrementos de $1^{\circ}$ (um grau) a $360^{\circ}$ (trezentos e sessenta graus), progressivos e regressivos de fácil visualização. As vantagens da utilização do flexímetro, segundo [Clarkson and Gilewich 1991], em relação a um goniômetro universal são: não é necessário alinhar o flexímetro com o eixo articular; os movimentos rotacionais e a avaliação do tronco são medidos com maior facilidade; ocorre pouca mudança no alinhamento do aparelho segundo os fabricantes.

O inclinômetro digital é um instrumento de funcionamento dependente da gravidade através de um sensor que capta medida da angulação. É um instrumento útil de fácil utilização, no entanto pouco difundido na fisioterapia com um custo mais elevado e independe de pontos anatômicos como o goniômetro universal [Bennell et al. 1998, de Winter et al. 2004].

O eletrogoniômetro é um instrumento relativamente novo com maiores possibili-

\footnotetext{
${ }^{1}$ Quantidade de movimento passiva ou ativa que paciente consegue realizar [Amado and Maria 2006]
} 
dades de aplicabilidade uma vez que pode-se realizar mensurações consecutivas, como no caso do FlexMeter, gerando um relatório em tempo real da sessão. Assim pode-se alterar a conduta ao visualizar este resultado beneficiando o paciente em questão. Alguns dos eletrogoniômetros existentes apenas mensuram a ADM naquele instante, o tempo de realização do movimento.

Por fim, o produto BioFeed [BioSmart 2011] é um equipamento que propõe monitorar movimentos do corpo humano, através da mensuração da inclinação dos membros do corpo em relação ao solo. Contudo, informações técnicas não estão disponíveis.

\section{FlexMeter}

O sistema FlexMeter [Polizel et al. 2011] tem como objetivo o acompanhamento e a supervisão do tratamento de pacientes de fisioterapia. Através de RSSF, mede-se ângulos articulares de um paciente ao longo de uma sessão de fisioterapia e exibe-se estes dados de três formas: quantitativamente, através do valor numérico e de gráficos em função do tempo, e qualitativamente, através de um modelo de computação gráfica do paciente, como observado na Figura 1. Dados característicos de cada sessão, tais como O valor máximo, mínimo e médio de cada ângulo medido, são armazenados após o término da sessão. Estes podem ser consultados e analisados, tanto individualmente a cada sessão como através de um gráfico composto por valores de várias sessões.

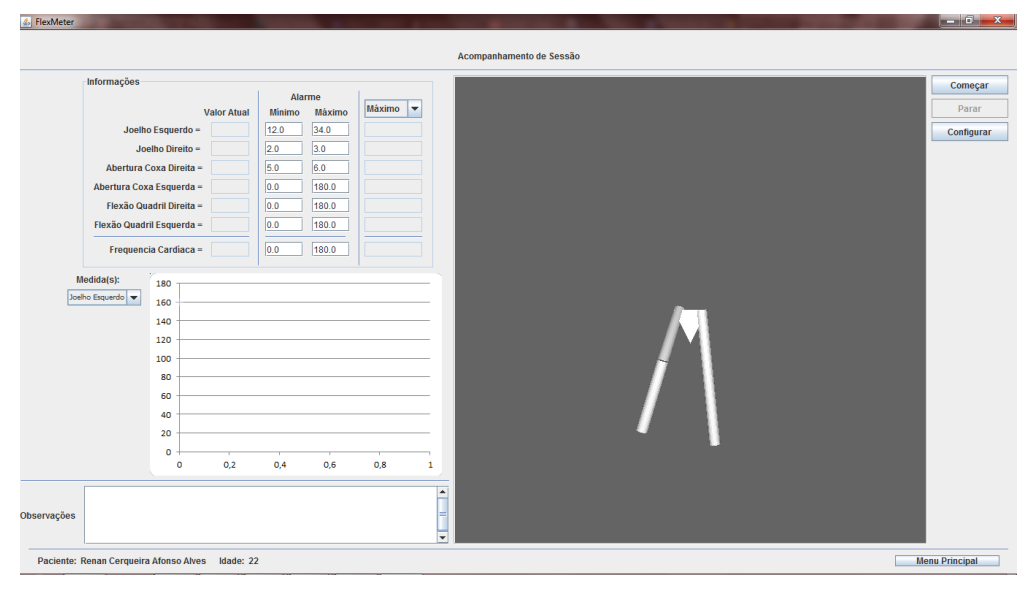

Figura 1. FlexMeter - interface

A Figura 2 exibe a arquitetura do sistema, indicando o posicionamento dos seis módulos sensores no paciente e a conexão do módulo sorvedouro com a estação do fisioterapeuta, onde o software de processamento de dados é executado. No protótipo construído foram contemplados somente ângulos articulares relativos a membros inferiores.

As subseções seguintes descrevem o procedimento utilizado para a realização dos cálculos de ângulos e os componentes de hardware e software que compõem o sistema.

\subsection{Procedimento de cálculos de ângulos}

$\mathrm{O}$ ângulo $\theta$ formado por dois vetores, $v_{1}$ e $v_{2}$, em espaço euclidiano pode ser calculado a partir de suas coordenadas em uma base ortonormal da seguinte forma:

$$
\theta=\arccos \left(\frac{v_{1} \cdot v_{2}}{\left|v_{1}\right|\left|v_{2}\right|}\right)
$$




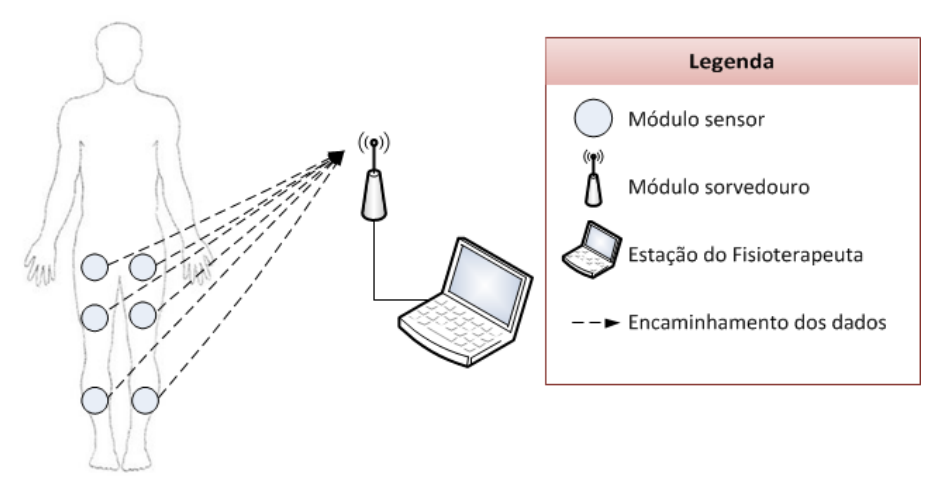

Figura 2. Arquitetura do Sistema

Assim, para determinar ângulos articulares de um paciente, basta determinar vetores que correspondam as linhas de mensuração dos ângulos em uma base comum. Estes vetores podem ser determinados através de módulos sensores acoplados ao paciente.

Para que seja possível o cálculo da direção de um sensor no espaço, algumas premissas são consideradas:

- a base $B=\left(b_{x}, b_{y}, b_{z}\right)$ do ambiente, comum a todos os módulos sensores, é fixa. Convenciona-se que $b_{z}$ é um vetor unitário no sentido da gravidade, $b_{x}$ é um vetor horizontal unitário perpendicular ao primeiro e $b_{y}$ é obtido através do produto vetorial dos primeiros;

- os módulos sensores possuem uma base ortogonal intrínseca variável em relação a base do ambiente. Suas medidas são feitas em relação a esta base;

- os módulos sensores possuem dispositivos para aferir as componentes da base do ambiente $\left(b_{z}\right.$ e $\left.b_{x}\right)$ em sua base intrínseca;

- algum dos eixos da base dos sensores deve estar alinhado com o ponto anatômico a ser observado. Convencionamos que seja o eixo X.

Desta forma, o problema do cálculo da direção de um sensor no espaço se resume em calcular a direção do eixo $\mathrm{X}$ da base dos sensores na base do ambiente.

Sejam $u=\left(u_{x}, u_{y}, u_{z}\right)$ e $v=\left(v_{x}, v_{y}, v_{z}\right)$ os vetores normalizados que representam os valores medidos por um módulo sensor de $b_{x}$ e $b_{z}$. Portanto, $v$ representa a direção da gravidade, enquanto que $u$ representa o vetor de referência horizontal, ambos representados na base do módulo sensor.

A matriz de mudança de base $M_{s a}$ é a matriz que, se multiplicada pela direita por um vetor, transforma suas coordenadas da base do ambiente para coordenadas na base do módulo sensor. Desta forma, a Equação 2 relaciona a referência horizontal da base do ambiente ao vetor $u$. Analogamente, a Equação 3 relaciona o vetor unitário na direção da gravidade ao valor aferido pelo módulo sensor. Por fim, a Equação 4 os respectivos produtos vetoriais $^{2}$ (a Equação 5 exibe o produto vetorial por extenso).

\footnotetext{
${ }^{2} \times$ é o sinal de produto vetorial
} 


$$
\begin{aligned}
& M_{s a} \cdot(1,0,0)=\left(u_{x}, u_{y}, u_{z}\right) \\
& M_{s a} \cdot(0,0,1)=\left(v_{x}, v_{y}, v_{z}\right) \\
& M_{s a} \cdot(0,1,0)=\left(v_{y} \cdot u_{z}-v_{z} \cdot u_{y}, v_{z} \cdot u_{x}-v_{x} \cdot u_{z}, v_{x} \cdot u_{y}-v_{y} \cdot u_{x}\right) \\
& u \times v=\left(v_{y} \cdot u_{z}-v_{z} \cdot u_{y}, v_{z} \cdot u_{x}-v_{x} \cdot u_{z}, v_{x} \cdot u_{y}-v_{y} \cdot u_{x}\right)
\end{aligned}
$$

Com estas informações é possível construir a matriz $M_{s a}$, conforme Equação 6 . Tendo $M_{s a}$ em mãos, o vetor correspondente a direção do eixo X do módulo sensor pode ser calculada pela Equação 7.

$$
\begin{gathered}
M_{s a}=\left[\begin{array}{lll}
u_{x} & v_{y} \cdot u_{z}-v_{z} \cdot u_{y} & v_{x} \\
u_{y} & v_{z} \cdot u_{x}-v_{x} \cdot u_{z} & v_{y} \\
u_{z} & v_{x} \cdot u_{y}-v_{y} \cdot u_{x} & v_{z}
\end{array}\right] \\
M_{s a} \cdot(x, y, z)=(1,0,0)
\end{gathered}
$$

Resolvendo o sistema obtêm-se os valores $x, y$ e $z$ correspondentes às coordenadas do vetor unitário na direção do eixo $\mathrm{X}$ do módulo sensor na base do ambiente. Assim, para calcular o ângulo entre dois módulos sensores, determina-se a direção do eixo $\mathrm{X}$ de ambos na base do ambiente e calcula-se o ângulo com a equação 1 .

Para o protótipo apresentado, o sensor utilizado para aferir $b_{z}$ foi o acelerômetro. A calibração de cada um de seus eixos foi realizada através da aquisição de valores simétricos conhecidos, desta forma o offset do sensor é dado pela média aritmética de todos os valores adquiridos [Shimmer Research 2011a].

Dado o uso exclusivo de acelerômetro, não foi utilizado nenhum sensor para aferir o vetor de referência horizontal, tendo sido adotado um valor constante. Devido a esta simplificação, o sistema é capaz de aferir ângulos articulares somente nos casos em que o eixo $\mathrm{X}$ dos módulos sensores encontram-se em um mesmo plano vertical. Esta restrição pode ser contornada a partir da adição de outros sensores, como magnetômetros e giroscópios.

\subsection{Hardware}

Dos sete módulos sensores do sistema, seis são módulos básicos Shimmer [Shimmer Research 2011b], vistos na Figura 3. Estes módulos são munidos de acelerômetro de três eixos e bateria. O sétimo módulo é um telosB [MEMSIC Inc. 2004] que, no escopo do sistema, tem a função de sorvedouro de dados, ou seja, receptor dos dados da aplicação.

Ambos tipos de módulos seguem o padrão de comunicação sem fio IEEE 802.15.4 [IEEE Standard 2006], implementado pelo microcontrolador do rádio transceptor CC2420 [Texas Instruments 2007], que além do recursos de acesso ao meio, fornece serviços de segurança como confidencialidade, integridade e autenticidade dos dados, recurso importante para atender os requisitos de privacidade do paciente. 


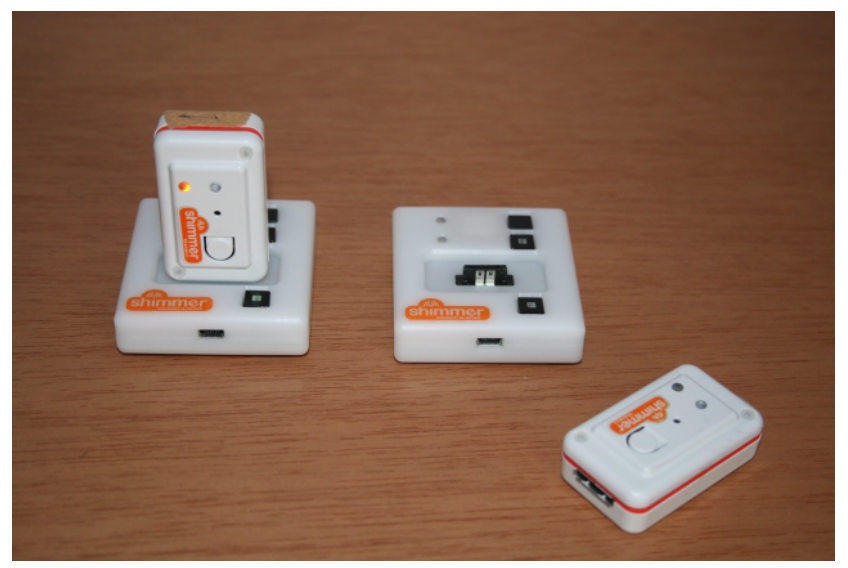

Figura 3. Módulos Sensores

\subsection{Sofware}

O software pode ser dividido em duas categorias: o software embarcado desenvolvido para os módulos sensores e o software da estação do fisioterapeuta, dividido em processamento de dados, interface gráfica e banco de dados.

\subsubsection{Software Embarcado}

Os módulos sensores utilizam o TinyOS [Levis et al. 2005], um sistema operacional dirigido a eventos projetado especialmente para plataformas com recursos de memória e processamento restritos. Um programa feito para TinyOS é composto pela instanciação de componentes e a interação destes com o programa principal através de suas interfaces.

Os componentes em geral representam componentes de hardware, como módulos de comunicação ou sensores, enquanto que as interfaces definem os comandos que os componentes podem realizar. A codificação é realizada em nesC [Gay et al. 2003], uma linguagem de programação derivada da linguagem $\mathrm{C}$.

O software desenvolvido para os módulos sensores pode ser dividido em três etapas de execução distintas: inicialização dos componentes, leitura e envio de valores.

A inicialização contempla a ativação do acelerômetro, do módulo de comunicação e de um componente de temporização responsável pelo disparo das leituras dos sensores. Após a inicialização do sistema embarcado, uma sequência periódica de leituras e envios de dados é mantida durante o uso do sistema.

A leitura do acelerômetro é composta pela obtenção de dados referente a três eixos ortogonais, formando uma tripla de valores inteiros de 16 bits. A partir da finalização de cada leitura, os dados obtidos são encapsulados e encaminhados à interface de rádio, que por sua vez os envia para o módulo sorvedouro.

\subsubsection{Software de Processamento de Dados}

O software que calcula os resultados a partir dos dados obtidos dos módulos sensores é composto pela arquitetura apresentada na Figura 4. 


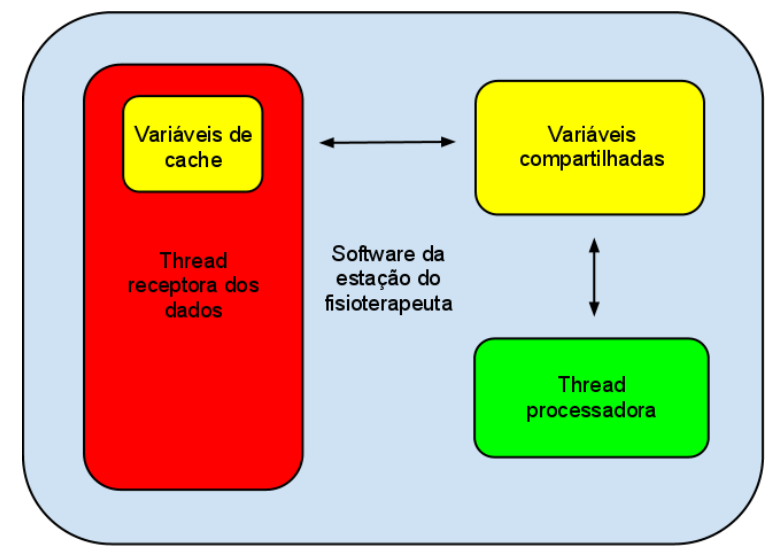

Figura 4. Arquitetura do software de processamento de dados

O software é composto basicamente por duas threads, além daquelas que controlam a interface gráfica com o usuário. Uma destas threads, intitulada "Thread Receptora", é responsável por receber os dados dos módulos sensores através do módulo sorvedouro. Além disso, é responsável por adicionar os dados recebidos ao log do sistema.

A outra thread, a "Thread Processadora", transforma os dados obtidos pela primeira, com base nos cálculos definidos da seção 3.1, e então submete os resultados para serem exibidos na interface gráfica. Esta também exerce a função de armazenar os valor máximo, mínimo e médio ao término da sessão.

\subsubsection{Banco de dados}

O sistema utiliza o gerenciador de bancos de dados MySQL [Oracle Corporation 2011] para armazenar os dados de pacientes e tratamentos. O banco de dados é composto por três tabelas: Paciente, Sessão e Medição. As informações armazenadas são dados pessoais do paciente, observações feitas pela fisioterapeuta e os valores sumarizados medidos durante cada uma das sessões.

\subsection{Modelo Tridimensional}

Para melhor visualização dos dados, foi construído um modelo 3D de paciente, que é exibido em um papplet na interface gráfica. Papplet é um componente do Processing, um ambiente de desenvolvimento de aplicações 3D, integrável com aplicações Java.

Tal modelo consiste de quatro cilindros, que representam os grandes ossos da perna, e um triângulo, que representa a quadril. Estes objetos são posicionados na tela de acordo com as posições calculadas dos dados dos módulos sensores, como na Figura 1.

O modelo possui as seguintes funções auxiliares de visualização: zoom através do botão de rolagem do mouse; destaque de membros que formam determinados ângulos e reposicionamento da câmera com arrasto do botão direito do mouse.

\section{Resultados}

Nesta seção serão apresentados os resultados obtidos com o desenvolvimento do sistema. Estes contemplam o processo de validação das mensurações e da interface. 


\subsection{Validação das Medidas do FlexMeter}

Com objetivo de averiguar a pertinência das medidas do sistema, comparou-se sua saída com ângulos estáticos determinados por um goniômetro. Quatro ângulos foram escolhidos arbitrariamente dentro do intervalo $\left[0^{\circ}, 140^{\circ}\right]$, intervalo esperado típico para uma articulação de joelho [Marques 2003].

Dois módulos sensores foram posicionados um em cada braço do goniômetro, de forma que o ângulo entre os módulos sensores fosse o mesmo que o indicado pelo instrumento.

O valor retornado pelo sistema foi coletado 20 vezes para cada um dos quatro ângulos de referência escolhidos $\left(0^{\circ}, 45^{\circ}, 90^{\circ}\right.$ e $\left.120^{\circ}\right)$. Parte do erro existente nestas medidas é proveniente do erro inerente ao goniômetro e do desalinhamentos da posição relativa entre os módulos sensores e os braços do goniômetro.

Com estes dados é possível estabelecer o intervalo de confiança para cada mensuração de ângulo. Utilizando o nível de confiança de $95 \%$, assumindo que as medidas estão distribuídas de acordo com uma distribuição normal e utilizando equações clássicas obtemos a Tabela 1.

Tabela 1. Valores de mensuração do sistema - estatísticas

\begin{tabular}{|c|c|c|c|c|}
\hline \multicolumn{1}{c|}{} & \multicolumn{4}{|c|}{ Ângulos de Referência } \\
\hline Estatística & $0^{\circ}$ & $45^{\circ}$ & $90^{\circ}$ & $120^{\circ}$ \\
\hline Média & $1.695^{\circ}$ & $45.381^{\circ}$ & $90.429^{\circ}$ & $120.079^{\circ}$ \\
Comprimento do intervalo de confiança & $0.445^{\circ}$ & $0.960^{\circ}$ & $0.832^{\circ}$ & $0.695^{\circ}$ \\
Erro máximo no intervalo de confiança & $2.14^{\circ}$ & $1.34^{\circ}$ & $1.26^{\circ}$ & $0.77^{\circ}$ \\
\hline
\end{tabular}

Para considerar o erro do goniômetro na estimativa do erro do sistema, utilizase as equações 8 e 9, para a determinação do erro médio e do desvio do erro médio, respectivamente. Desta forma, o valor pontual do erro do sistema é dado pela diferença entre o valor apontado pelo goniômetro e a média dos valores apontados pelo sistema.

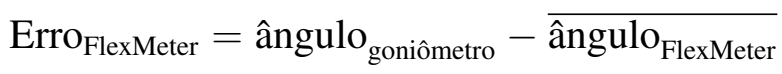

$$
\begin{aligned}
& \sigma_{\text {Erro }_{\text {FlexMeter }}}=\sqrt{\sigma_{\text {ângulo goniômetro }}^{2}+\sigma_{\text {ângulo }}^{2} \text { FlexMeter }}
\end{aligned}
$$

Foi considerado que o erro do goniômetro, $\sigma_{\text {ângulo }}{ }_{\text {goniômetro }}$, é igual a metade da menor divisão do instrumento, isto é, $1^{\circ}$. Para o erro do sistema, utilizou-se o desvio padrão da amostra, de acordo com o ângulo considerado.

Os resultados dos cálculos do erro do sistema e seu desvio encontram-se na tabela 2. O erro médio é pequeno, porém seu desvio é grande. Contudo, de acordo com a Tabela 1 , observa-se que o erro médio não é muito maior do que $2^{\circ}$, com $95 \%$ de confiança.

\subsection{Usabilidade}

Com a finalidade de avaliar a usabilidade do sistema, a fisioterapeuta co-autora utilizou o protótipo em ambiente clínico. Após a leitura da versão preliminar do manual de 


Tabela 2. Valores de erro do sistema
\begin{tabular}{|c|c|c|}
\hline Ângulo & Erro & Desvio do Erro \\
\hline $0^{\circ}$ & $1.7^{\circ}$ & $1.4^{\circ}$ \\
$45^{\circ}$ & $0.4^{\circ}$ & $2.4^{\circ}$ \\
$90^{\circ}$ & $0.4^{\circ}$ & $2.1^{\circ}$ \\
$120^{\circ}$ & $0.1^{\circ}$ & $1.9^{\circ}$ \\
\hline
\end{tabular}

usuário e as funções fornecidas pelo sistema foram testadas. Notou-se a falta de algumas explicações básicas, porém necessárias para um usuário que não havia entrado em contato com o sistema anteriormente

Quanto ao protocolo de uso, trecho do manual que indica ao seu leitor como obter a medida dos ângulos desejados, o manual trouxe os procedimentos necessários. Por exemplo, para realizar a mensuração do ângulo de flexão ou extensão da articulação do joelho, o manual instrui o fisioterapeuta a posicionar dois módulos sensores, respectivamente, no músculo reto femural e músculo tibial anterior do paciente.

No caso desta articulação, é importante notar que ao usar o goniômetro universal o posicionamento deveria ser no plano sagital entre os côndilos femural e tibial e o terapeuta teria que manipular o instrumento para acompanhar o movimento.

Porém, foi preciso explicitar no manual que alguns ângulos, como os de flexão e extensão do joelho, são medidos com o mesmo par de módulos sensores e a diferenciação entre eles fica a cargo do usuário.

Não foram observados bugs ou comportamentos inesperados durante a operação. Após a compreensão dos pontos deficientes do manual, o usuário foi capaz de utilizar o sistema, seguir as etapas de preparação e realizar a mensuração do ângulo de flexão do joelho direito e observar os dados obtidos.

Portanto, os resultados obtidos através do processo de validação de mensuração dos ângulos e a avaliação de usabilidade realizada pela fisioterapeuta demostram o potencial do sistema como aplicação prática.

\section{Considerações Finais}

No contexto das cidades inteligentes, desenvolver ferramentas de apoio a fisioterapia permite expandir o suporte a área de saúde. Este trabalho é resultado da intersecção entre a engenharia e fisioterapia, proporcionando maior automação e poder de processamento de dados na tarefa de monitoramento de amplitude de movimento através do sistema proposto.

O protótipo descrito e validado neste artigo realiza a mensuração de ângulos articulares em tempo real, através de dispositivos de sensoriamento conectados por redes redes sem fio, garantindo a mobilidade do paciente e fisioterapeuta. O padrão de rede sem fio utilizado é o 802.15.4 [IEEE Standard 2006], apropriado para aplicações que possuem requisitos relacionados à consumo de energia e privacidade.

Os dados que o sistema armazena durante as sessões permite ao fisioterapeuta acompanhar a evolução do paciente e gerar relatórios clínicos completos, permitindo quantificar a efetividade do tratamento. 
O processo de validação constatou que o erro cometido pelo sistema ao realizar mensurações de ângulos articulares é da mesma ordem de grandeza de goniômetro. $\mathrm{O}$ teste de usabilidade realizado contemplou a implantação preliminar do sistema em ambiente clínico. Com estas informações, conclui-se que o sistema possui potencial para se tornar uma aplicação prática.

Como discutido na seção 3.1, a adição de outros sensores, como magnetômetros e giroscópios, permitirão ao sistema aferir ângulos articulares em qualquer posição do paciente. Neste caso, o sistema poderia ser utilizado como ferramenta de suporte e acompanhamento em educação física, após a adição de filtros para lidar com as grandes acelerações envolvidas. Além disso, pretende-se estender os testes realizados, reproduzindo o experimento em diversos pacientes e com maior número de fisioterapeutas.

\section{Agradecimentos}

Bruno Trevizan de Oliveira possui Bolsa de Mestrado financiada pela Fundação de Amparo à Pesquisa do Estado de São Paulo, processo FAPESP 2010/02909-8.

\section{Referências}

Amado, J. and Maria, S. (2006). Métodos de Avaliação cínica e funcional em fisioterapia. Guanabara Koogan.

Bennell, K., Talbot, R., Wajswelner, H., Techovanich, W., Kelly, D., and Hall, A. (1998). Intra-rater and inter-rater reliability of a weight-bearing lunge measure of ankle dorsiflexion. Aust J Physiother, 44(3):175-180.

BioSmart (2011). Biofeed. http://www.biosmart.com.br/biofeed.html.

Clarkson, H. and Gilewich, G. (1991). Avaliaçao musculo-esqueletica: amplitude de movimento articular e força muscular manual. Manole.

COFFITO (2012). Definição de fisioterapia. Conselho Federal de Fisioterapia e Terapia Ocupacional.

Culler, D., Estrin, D., and Srivastava, M. (2004). Overview of sensor networks. Computer Magazine, 37(8):41-49.

de Winter, A. F., Heemskerk, M. A. M. B., Terwee, C. B., Jans, M. P., Devillé;, W., van Schaardenburg, D.-J., Scholten, R. J. P. M., and Bouter, L. M. (2004). Inter-observer reproducibility of measurements of range of motion in patients with shoulder pain using a digital inclinometer. BMC Musculoskelet Disord, 5:18.

Gajdosik, R. L. and Bohannon, R. W. (1987). Clinical measurement of range of motion. review of goniometry emphasizing reliability and validity. Phys Ther, 67(12):18671872 .

Gay, D., Welsh, M., Levis, P., Brewer, E., Behren, R. V., and Culler, D. (2003). The nesc language: A holistic approach to networked embedded systems. In In Proceedings of Programming Language Design and Implementation (PLDI, pages 1-11.

IEEE Standard (2006). IEEE 802.15.4: Wireless medium access control (MAC) and physical layer (PHY) specifications for low-rate wireless personal area networks (WPANs). 
Levis, P., Madden, S., Polastre, J., Szewczyk, R., Whitehouse, K., Woo, A., Gay, D., Hill, J., Welsh, M., Brewer, E., and Culler, D. (2005). TinyOS: an operating system for sensor networks ambient intelligence. In Weber, W., Rabaey, J. M., and Aarts, E., editors, Ambient Intelligence, chapter 7, pages 115-148. Springer Berlin Heidelberg, Berlin/Heidelberg.

Lorincz, K., Chen, B.-r., Challen, G. W., Chowdhury, A. R., Patel, S., Bonato, P., and Welsh, M. (2009). Mercury: a wearable sensor network platform for high-fidelity motion analysis. In Proceedings of the 7th ACM Conference on Embedded Networked Sensor Systems, SenSys '09, pages 183-196, New York, NY, USA. ACM.

Malan, D., Fulford-jones, T., Welsh, M., and Moulton, S. (2004). Codeblue: An ad hoc sensor network infrastructure for emergency medical care. In In International Workshop on Wearable and Implantable Body Sensor Networks.

Marques, A. (2003). Manual de goniometria. Manole, segunda edition.

MEMSIC Inc. (2004). telosb product details - 6020-0094-04 rev b. http://www.memsic.com/support/documentation/wireless-sensornetworks/category/7-datasheets.html?download $=152$

O'Donovan, K. and Ayer, S. (2011). Real-time joint angle measurement using the shimmer wireless sensor platform. In Proceedings of the First ACM Workshop on Mobile Systems, Applications, and Services for Healthcare, mHealthSys '11, pages 7:1-7:2, New York, NY, USA. ACM.

O'donovan, K., Kamnik, R., O'keeffe, D., and Lyons, G. (2007). An inertial and magnetic sensor based technique for joint angle measurement. J Biomech, 40(12):2604-11.

Oracle Corporation (2011). Mysql website. http://www.mysql.com/.

Polizel, A. S., Wada, E. D., and Alves, R. C. A. (2011). Redes de sensores sem fio aplicadas à fisioterapia. Tese de conclusão de curso, Escola Politécnica, Universidade de São Paulo, São Paulo.

Portney, L. and Watkins, M. (2000). Foundations of clinical research: applications to practice. Prentice Hall Health.

Rothstein, J. (1985). Measurement in physical therapy. Clinics in physical therapy. Churchill Livingstone.

Shimmer Research (2011a). 9DOF calibration user manual - Rev 0.1b. http://www.shimmer-research.com/wp-content/uploads/2011/06/Shimmer-9DOFCalibration-Application-User-Manual-Rev0.1b.pdf.

Shimmer Research (2011b). Shimmer - wireless sensor platform. http://www.shimmerresearch.com/wp-content/uploads/2011/05/Shimmer-Platform.pdf.

Silva, H., Afonso, J., Morim, P., Oliveira, P., Correia, J., and Rocha, L. (2007). Wireless hydrotherapy smart-suit network for posture monitoring. In Industrial Electronics, 2007. ISIE 2007. IEEE International Symposium on, pages 2713-2717.

Texas Instruments (2007). Chipcon cc2420 datasheet. http://inst.eecs.berkeley.edu/ cs150/Documents/CC2420.pdf. 